\title{
Is staged bilateral thalamic radiosurgery an option for otherwise surgically ineligible patients with medically refractory bilateral tremor?
}

\author{
Ajay Niranjan, MD, MBA, ${ }^{1}$ Sudesh S. Raju, BA, ${ }^{2}$ Edward A. Monaco III, MD, PhD, ${ }^{1}$ \\ John C. Flickinger, MD, ${ }^{3}$ and L. Dade Lunsford, MD'
}

Departments of ${ }^{1}$ Neurological Surgery and ${ }^{3}$ Radiation Oncology, University of Pittsburgh Medical Center; and ${ }^{2}$ University of Pittsburgh School of Medicine, Pittsburgh, Pennsylvania

OBJECTIVE Unilateral Gamma Knife thalamotomy (GKT) is a well-established treatment for patients with medically refractory tremor who are not eligible for invasive procedures due to increased risk of compications. The purpose of this study was to evaluate whether staged bilateral GKT provides benefit with acceptable risk to patients suffering from disabling medically refractory bilateral tremor.

METHODS Eleven patients underwent staged bilateral GKT during a 17-year period (1999-2016). Eight patients had essential tremor (ET), 2 had Parkinson's disease (PD)-related tremor, and 1 had multiple-sclerosis (MS)-related tremor. For the first GKT, a median maximum dose of $140 \mathrm{~Gy}$ was delivered to the posterior-inferior region of the nucleus ventralis intermedius (VIM) through a single isocenter with 4-mm collimators. Patients who benefitted from unilateral GKT were eligible for a contralateral GKT 1-2 years later (median 22 months). For the second GKT, a median maximum dose of $130 \mathrm{~Gy}$ was delivered to the opposite VIM nucleus to a single 4-mm isocenter. The Fahn-Tolosa-Marin (FTM) clinical tremor rating scale was used to score tremor, drawing, and drinking before and after each GKT. The FTM writing score was assessed only for the dominant hand before and after the first GKT. The Karnofsky Performance Status (KPS) was used to assess quality of life and activities of daily living before and after the first and second GKT.

RESULTS The median time to last follow-up after the first GKT was 35 months (range 11-70 months). All patients had improvement in at least 1 FTM score after the first GKT. Three patients $(27.3 \%)$ had tremor arrest and complete restoration of function (noted via FTM tremor, writing, drawing, and drinking scores equaling zero). No patient had tremor recurrence or diminished tremor relief after the first GKT. One patient experienced new temporary neurological deficit (contralateral lower-extremity hemiparesis) from the first GKT. The median time to last follow-up after the second GKT was 12 months (range 2-70 months). Nine patients had improvement in at least 1 FTM score after the second GKT. Two patients had tremor arrest and complete restoration of function. No patient experienced tremor recurrence or diminished tremor relief after the second GKT. No patient experienced new neurological or radiological adverse effect from the second GKT. Statistically significant improvements were noted in the KPS score following the first and second GKT.

CONCLUSIONS Staged bilateral GKT provided effective relief for medically refractory, disabling, bilateral tremor without increased risk of neurological complications. It is an appropriate strategy for carefully selected patients with medically refractory bilateral tremor who are not eligible for deep brain stimulation.

https://thejns.org/doi/abs/10.3171/2016.11.JNS162044

KEY WORDS stereotactic radiosurgery; Gamma Knife; thalamotomy; bilateral; essential tremor; Parkinson's disease; multiple sclerosis; tremor; movement disorder; functional neurosurgery

$\mathrm{T}$ REMOR is the most common movement disorder across the world, with more than 5 million individuals affected in the United States alone..$^{1,18}$ Many patients suffer from bilateral tremor. Over time medical therapy may fail to control tremor for these patients, and consideration of surgical intervention may ultimately be warranted. Surgical options available include deep brain stimulation (DBS) and radiofrequency thalamotomy (RFT). While bilateral DBS and bilateral RFT provide tremor improvement, they are not feasible in patients with

ABBREVIATIONS AC = anterior commissure; $A E$ = adverse event; $A$ RE = adverse radiation effect; DBS = deep brain stimulation; ET = essential tremor; FTM = Fahn-Tolosa-Marin; GKT = GK thalamotomy; IC = internal capsule; KPS = Karnofsky Performance Status; MRgFUS = magnetic resonance-guided focused ultrasound; MS = multiple sclerosis; $\mathrm{PC}=$ posterior commissure; $\mathrm{PD}=$ Parkinson's disease; $\mathrm{RFT}=$ radiofrequency thalamotomy; VIM = nucleus ventralis intermedius.

SUBMITTED August 5, 2016. ACCEPTED November 17, 2016.

INCLUDE WHEN CITING Published online April 7, 2017; DOI: 10.3171/2016.11.JNS162044. 
coagulation disorders, and in rare cases they are associated with complications such as dysphagia, dysarthria, gait ataxia, and hypophonia. ${ }^{14,26}$ Gamma Knife thalamotomy (GKT) is especially valuable for patients ineligible for such invasive procedures. ${ }^{5,20,22,35,37}$ The purpose of this study was to evaluate the clinical benefits and potential adverse effects in patients who underwent staged bilateral GKT for tremor.

\section{Methods}

\section{Patient Selection Evaluation}

We reviewed the medical and imaging records of 11 patients (6 male and 5 female) who underwent staged bilateral GKT during a 17-year period (March 1999-April 2016) (Table 1). This study was approved by the University of Pittsburgh institutional review board. Patients with insufficient response to medical therapy and ineligible for more invasive surgery were referred for consideration of GKT. The main reason for the second (staged bilateral) GKT was severe disabling bilateral tremor that markedly impaired occupations (in 3 cases involving a painter, a dentist, and a teacher) or activities of daily living (in 6 cases) or patient request following progression of tremor on the untreated side (in 2 cases).

Eight patients had essential tremor (ET), 2 patients had Parkinson's disease (PD)-related tremor, and 1 patient had multiple sclerosis (MS)-related tremor. The patients' mean age at their first GKT was 66.7 years. In 10 of 11 patients, the first GKT treated the dominant hand tremor. In the other patient, the first GKT treated the nondominant hand, which was more severely affected by tremor. Patients who had tremor reduction without a new neurological deficit were eligible for a contralateral second GKT after an observation period of 1 year. This duration of the observation period (at least 1 year after the first GKT) was used to assess tremor response in the treated arm (expected in $86 \%$ of patients at a median 4 months) and to evaluate the risk of new neurological deficit (expected in $4 \%$ at a median time of 7 months). ${ }^{16}$ The median time between the first and second GKT was 22 months (range 7-70 months). The mean age of the patients at the second GKT was 69.2 years. We used the Fahn-Tolosa-Marin (FTM) clinical tremor rating scale to score tremor, drawing, and drinking before and after each GKT. ${ }^{8}$ The FTM writing score was assessed only in the dominant hand before and after the first GKT. We also used the Karnofsky Performance Status (KPS) scores to grade quality of life before and after the first and second GKT. ${ }^{13}$

\section{GKT Technique}

The Leksell Model G stereotactic coordinate frame (Elekta Instruments) was attached to the patient's head, and high-resolution MR images were acquired via a 1.5- or 3.0-T scanner. The target was localized to the nucleus ventralis intermedius (VIM) of the thalamus via coordinates relative to the anterior commissure-posterior commissure (AC-PC) line. Specifically, the 1-mm-thick, gadoliniumenhanced MR slices from the spoiled-gradient recalled acquisition in steady state sequence were used to localize the target. Additional adjustments of target localization
TABLE 1. Clinical and demographic characteristics of patients included in this study

\begin{tabular}{|c|c|}
\hline Parameter & Value \\
\hline Total no. of pts & $11(100 \%)$ \\
\hline \multicolumn{2}{|l|}{ Age at radiosurgery (yrs) } \\
\hline \multicolumn{2}{|l|}{ 1st GKT } \\
\hline Median & 68 \\
\hline Range & $42-84$ \\
\hline \multicolumn{2}{|l|}{ 2nd GKT } \\
\hline Median & 73 \\
\hline Range & $44-84$ \\
\hline Family history of tremor & $9(81.8 \%)$ \\
\hline \multicolumn{2}{|l|}{ Dominant hand } \\
\hline Right & $9(81.8 \%)$ \\
\hline Left & $2(18.2 \%)$ \\
\hline \multicolumn{2}{|l|}{ Type of tremor } \\
\hline ET & $8(72.7 \%)$ \\
\hline PD & $2(18.2 \%)$ \\
\hline MS & $1(9.1 \%)$ \\
\hline \multicolumn{2}{|c|}{ Duration of tremor pre-GKT (yrs) } \\
\hline \multicolumn{2}{|l|}{ 1st GKT } \\
\hline Median & 6 \\
\hline Range & $3-60$ \\
\hline \multicolumn{2}{|l|}{ 2nd GKT } \\
\hline Median & 10 \\
\hline Range & $4-50$ \\
\hline \multicolumn{2}{|l|}{ Sex } \\
\hline Male & $6(54.5 \%)$ \\
\hline Female & $5(45.5 \%)$ \\
\hline \multicolumn{2}{|l|}{ Treatment side, 1st GKT } \\
\hline Left & $8(72.7 \%)$ \\
\hline Right & $3(27.3 \%)$ \\
\hline
\end{tabular}

Pts $=$ patients.

Data are presented as number of patients (\%) unless otherwise indicated.

were made in respect to the internal capsule (IC) as shown on axial and coronal fast inversion recovery images. Final target coordinates were further validated by means of GammaPlan software (Elekta Instruments).

The target was localized in the following stages: $50 \%$ of the width of the third ventricle plus $11 \mathrm{~mm}$ lateral to the AC-PC line (x coordinate); $25 \%$ of the AC-PC distance (+ 1 or $2 \mathrm{~mm}$ ) anterior to the PC (y coordinate); and a radiosurgery isocenter ( $4 \mathrm{~mm}$ collimator) $2 \mathrm{~mm}$ superior to the AC-PC line (z coordinate). The inferior border of the radiosurgery lesion using the 4-mm collimator of the Leksell Gamma Knife (Elekta $\mathrm{AB}$ ) was projected to extend just inferior to the AC-PC line. The lateral edge of the isocenter (x coordinate) was adjusted based on the location of the IC. The $30 \%$ isodose line was placed medial to the IC. During the utilization of Model B, C, 4C, or Perfexion Gamma Knife, selective beam or sector blocking was used to reduce the dose delivered to the IC. For the Model $\mathrm{U}$ unit, which has a sharper fall-off of radiation in the $\mathrm{x}$ plane, no beam blocking was used. 


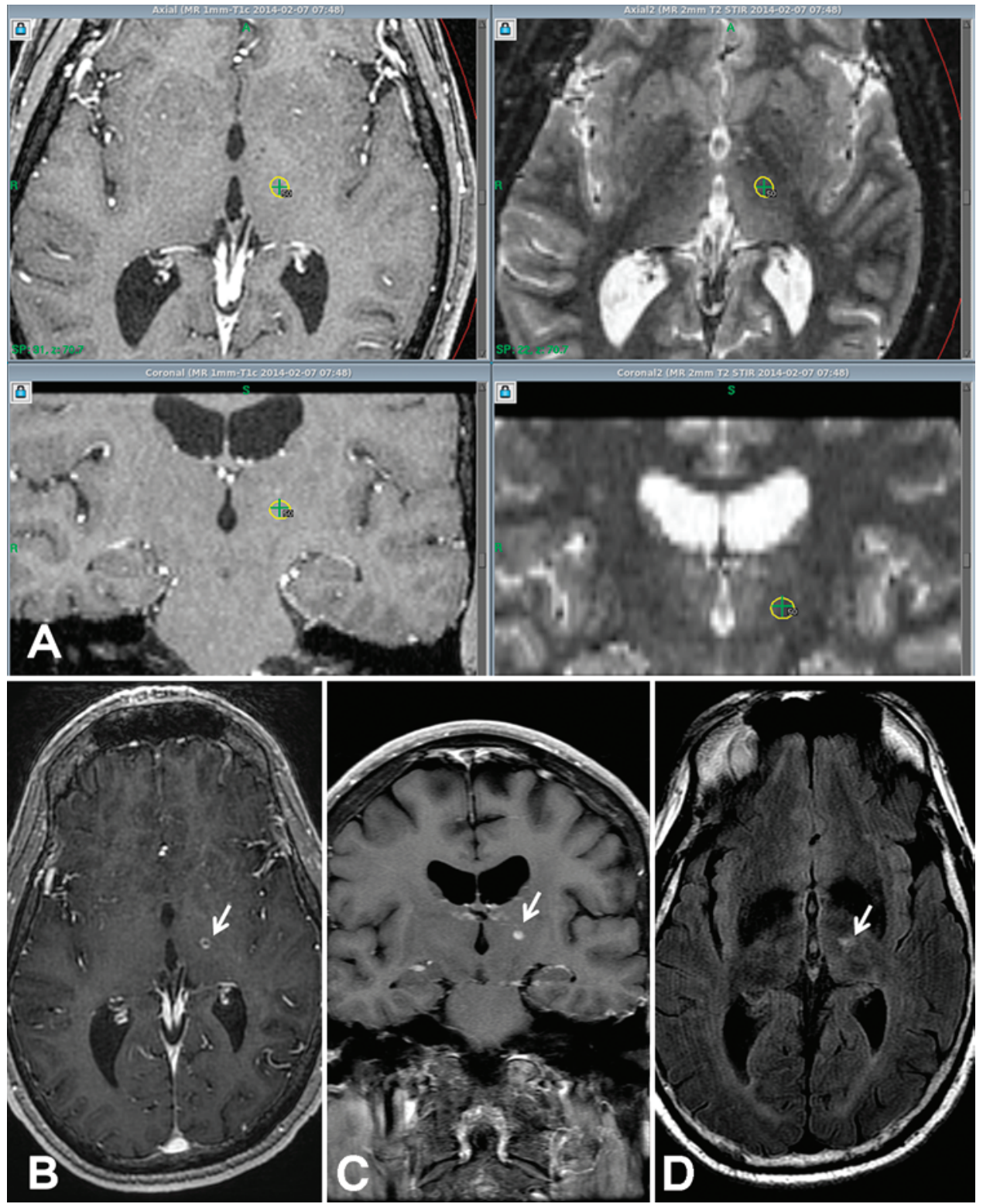

FIG. 1. A: GammaPlan screenshot showing dose planning (yellow circles) and target selection for first (left VIM) GKT. B-D: Contrast-enhanced axial T1-weighted (B) and coronal (C) and axial (D) T2-weighted FLAIR images obtained 6 months after first (left VIM) GKT showing enhancing left thalamic lesion (solid arrows). Figure is available in color online only.

The median dose for first GKT in this group was 140 Gy. At our institution the central dose varied from 130 Gy to 140 Gy (Fig. 1). However, 2 patients had their first GKT at outside institutions where high central doses were used (200 Gy and $150 \mathrm{~Gy})$. These doses were delivered to the posterior-inferior region of the VIM through a single 4-mm isocenter. For the second GKT, a median dose of 130 Gy (range 120-140 Gy) was delivered to the opposite posterior-inferior region of the VIM through a single 4-mm isocenter (Fig. 2).

\section{Follow-Up Evaluations}

Patients underwent clinical and radiological followup at 4- to 6-month intervals. We used the FTM clinical tremor rating scale to score tremor, drawing, and drinking (for both hands) after each GKT. We used the KPS scores to grade quality of life. Patients were also evaluated for any adverse event (AE), which was defined as a new neurological symptom or sign. Patients were also assessed for dysphagia, dysarthria, speech disorders, and gait ataxia. MR images were reviewed and the findings were categorized into 3 groups: enhancing lesion only, enhancing lesion with streaking along the IC, or expanded lesion with reactive changes and edema.

\section{Statistical Analysis}

The Wilcoxon signed-rank test was used to determine statistical significance of improvements in FTM values (tremor, handwriting, drawing, and drinking) and KPS scores following each GKT. The Wilcoxon Mann-Whit- 


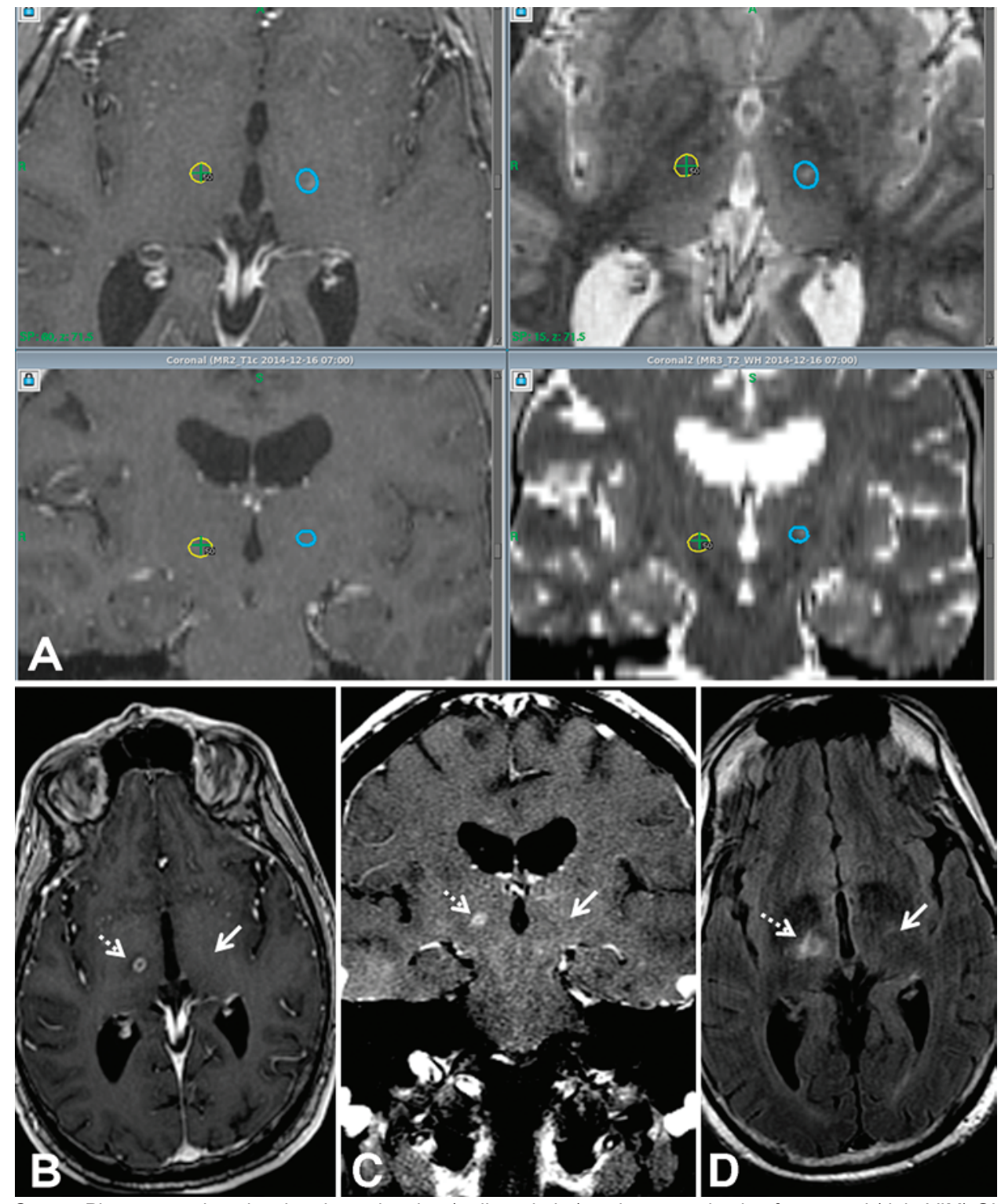

FIG. 2. A: GammaPlan screenshot showing dose planning (yellow circles) and target selection for second (right VIM) GKT. The blue circles show the volume that was irradiated at the first (left VIM) GKT. B-D: Contrast-enhanced axial T1-weighted (B) and coronal (C) and axial (D) T2-weighted FLAIR images obtained 6 months after second (right VIM) GKT. The right thalamic enhancing lesion (dotted arrows) is clearly seen, but the left thalamic lesion is only faintly visible (solid arrows). Figure is available in color online only.

ney U-test was used to determine statistical significance of differences between FTM values for pre-GKT (first vs second procedure) and post-GKT (first vs second procedure). Kaplan-Meier analysis was used to determine time to onset of tremor relief. The log-rank test was used to determine statistically significant different Kaplan-Meier curves for first and second GKT. Univariate Cox regression was used to determine factors correlated to time to onset of tremor relief. Factors with statistically significant correlations in univariate Cox regression were selected for multivariate analysis. Logistic regression was used to determine statistically significant independent variables as predictors of clinical benefit and tremor arrest (complete relief of tremor). A p value of 0.05 was used for statistical significance.

\section{Results}

\section{Response to First GKT}

The median time to last follow-up after the first GKT was 35 months (range 11-70 months). All mean FTM values showed statistically significant improvement postGKT (Table 2). All patients had benefit in at least 1 FTM score from the first GKT. Specifically, 9 patients (81.8\%) had improvement in all 4 FTM scores, while 2 patients (18.2\%) had improvement in only 1 FTM score. Three patients $(27.3 \%)$ experienced tremor arrest a median of 8 months post-GKT (range 1-35 months). No patient experienced tremor recurrence or diminished tremor relief. One patient experienced an adverse radiation effect (ARE; Type C lesion), with contralateral lower-extremity hemi- 
TABLE 2. FTM and KPS scores

\begin{tabular}{|c|c|c|c|c|c|c|c|c|}
\hline \multirow[b]{2}{*}{ Variable } & \multicolumn{4}{|c|}{ 1st GKT } & \multicolumn{4}{|c|}{ 2nd GKT } \\
\hline & No. of Pts & Preop & Postop & $p$ Value & No. of Pts & Preop & Postop & $p$ Value \\
\hline FTM, tremor & $11(100 \%)$ & $3.27 \pm 0.79$ & $1.09 \pm 0.94$ & 0.003 & $11(100 \%)$ & $2.91 \pm 0.83$ & $1.36 \pm 0.92$ & 0.007 \\
\hline FTM, writing & $10(90.9 \%)$ & $3.10 \pm 0.74$ & $0.80 \pm 1.03$ & 0.006 & - & - & - & - \\
\hline FTM, drawing & $11(100 \%)$ & $3.27 \pm 0.79$ & $1.18 \pm 1.33$ & 0.007 & $11(100 \%)$ & $2.81 \pm 0.87$ & $1.55 \pm 1.04$ & 0.017 \\
\hline FTM, drinking & $11(100 \%)$ & $3.18 \pm 0.60$ & $1.09 \pm 1.38$ & 0.006 & $11(100 \%)$ & $2.91 \pm 0.70$ & $1.64 \pm 1.12$ & 0.01 \\
\hline KPS score & $11(100 \%)$ & $42.7 \pm 7.9$ & $64.5 \pm 15.7$ & 0.005 & $11(100 \%)$ & $61.8 \pm 13.3$ & $75.5 \pm 21.6$ & 0.016 \\
\hline
\end{tabular}

Values are mean \pm SD unless otherwise indicated.

paresis 8 months post-GKT. This patient was treated with corticosteroids and gradually recovered completely.

Two patients had their first GKT performed at an outside institution. One patient had a left GKT (150 Gy) and showed remarkable improvement in tremor starting 1 month postGKT, with a barely perceptible tremor at the last follow-up (68 months post-GKT). Another patient had a left GKT (200 Gy) that led to tremor arrest and complete functional recovery (starting 1 month post-GKT), which was maintained until last follow-up (70 months post-GKT). The median time to onset of benefit was 3 months (range 2 weeks-11 months). Based on Kaplan-Meier analysis, the median expected time was 3 months (95\% CI 0-7.046 months).

\section{Response to Second GKT}

The median time to last follow-up after the second GKT was 12 months (range 2-70 months). As for the first GKT, all mean FTM values showed statistically significant decreases post-GKT (Table 2). Nine patients (81.8\%) improved in at least 1 FTM score. The median time to onset of benefit was 3 months (range 1-6 months). Specifically, 7 patients had improvement in all 3 FTM scores, 1 patient had improvement in 2 FTM scores, 1 patient had improvement in 1 FTM score, and 2 patients $(18.2 \%)$ did not show improvement in any FTM score. Two patients (18.2\%) experienced tremor arrest at a median of 7.5 months (range 2-13 months) post-GKT. No patient experienced tremor recurrence or diminished tremor relief. No patient experienced any ARE or adverse event (AE) from the second GKT. One patient had the second GKT performed at an outside institution and had sustained tremor arrest 13 months post-GKT and no neurological AE.

The first GKT resulted in a greater change in FTM scores than the second GKT (Table 2 and Fig. 3). However, based on the Wilcoxon Mann-Whitney U-test, there was no statistically significant difference in any mean FTM value (pre- or post-GKT) between the first and second GKT. For the second GKT, no independent variable had a statistically significant correlation to the time of tremor relief using univariate or multivariate Cox regression. Based on Kaplan-Meier analysis, the median expected time to tremor relief was 4 months (95\% CI 2.75-5.25 months). Based on log-rank, there was no statistically significant difference between time to onset of tremor relief for the first and second GKT (Fig. 4). Based on logistic regression, no independent variable had a statistically significant prediction of clinical benefit or tremor arrest after the second GKT.

Additionally, based on KPS scores, staged bilateral
GKT resulted in statistically significant improvements in quality of life and activities of daily living following the first and second GKT (Table 2).

\section{Imaging After Bilateral GKT}

Short-term MRI follow-up (3-8 months) was available for 8 thalamotomy procedures. These changes were evaluated according to the classification of Ohye et al. ${ }^{21,23}$ Three types of lesions were noted (Fig. 5). In 6 of 8 patients, the lesions were classified as Type A (5-mm or smaller enhancing lesion on T1-weighted post-Gd images with no enhancing streak along the IC on T2-weighted FLAIR images); in 1 patient the lesion was classified as Type B (6- to 8-mm enhancing lesion with streaking along the IC on T2-weighted FLAIR images); and in 1 patient, who was considered a super-responder, the lesion was classified as Type $\mathrm{C}$ (an enhancing lesion $>10 \mathrm{~mm}$ on T1-weighted post-Gd images with an adjacent area of signal hyperintensity on T2 FLAIR images). The patient with the Type C lesion, who was undergoing GKT for MS-related tremor, experienced contralateral lower-extremity hemiparesis starting 8 months after the first GKT and was treated with corticosteroids. This patient did not develop any AE after the second GKT. One patient who had been previously treated with bilateral DBS without success underwent CT follow-up. Because of significant artifact due to the presence of bilateral DBS leads and electrodes, the CT quality was suboptimal for detecting the radiosurgical imaging response. One additional patient who had CT imaging showed an appropriate enhancing 5-mm lesion.

Medium-term imaging follow-up (10-30 months) was available in 4 cases. All showed some reduction of the initial lesion volume. Long-term imaging follow-up ( $\geq 5$ years) was available in 3 cases. For the super-responder patient, by 13 months MRI showed reduction in the enhancing lesion diameter to $7 \mathrm{~mm}$ and significant resolution of the perilesional T2 FLAIR signal change. A further long-term follow-up at 5 years showed a residual 4- to 5-mm T1 nonenhancing thalamic lesion. Two additional patients showed residual 4- to 5-mm T1 nonenhancing lesions without residual T2 signal change at 5 years. No patient developed radiation necrosis, cyst, or any other unexpected radiological change after second GKT.

\section{Discussion}

\section{Rationale for Bilateral GKT}

In eligible patients with bilateral tremor, bilateral DBS 


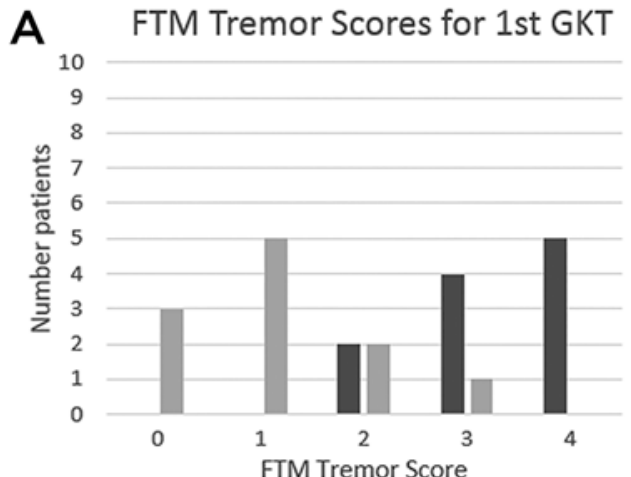

TM Drawing Scores for 1st GKT

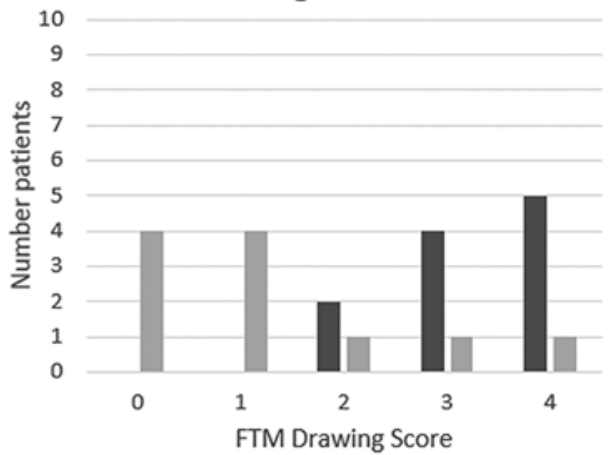

B FTM Tremor Scores for 2nd GKT

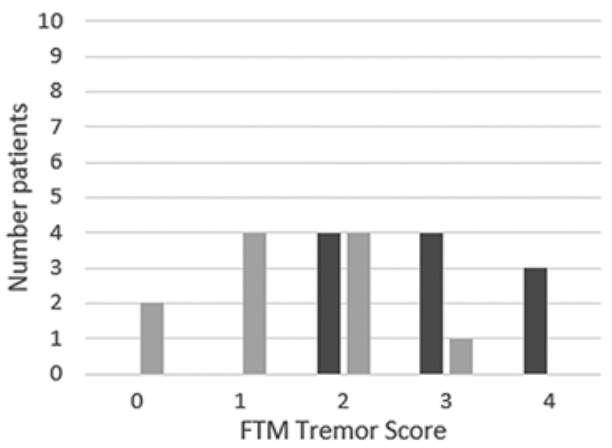

FTM Drinking Scores for 2nd GKT

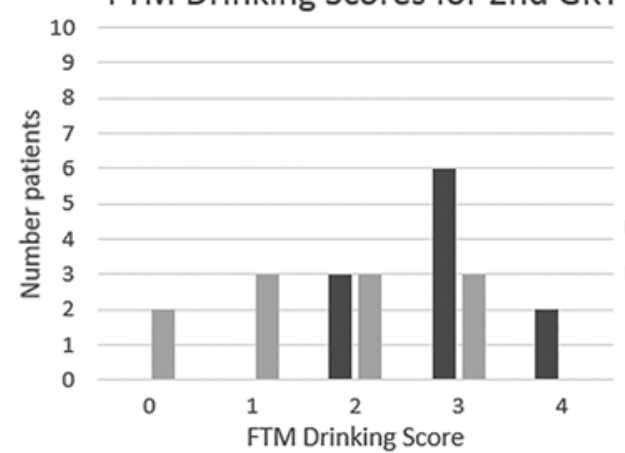

FTM Writing Scores for 1st GKT

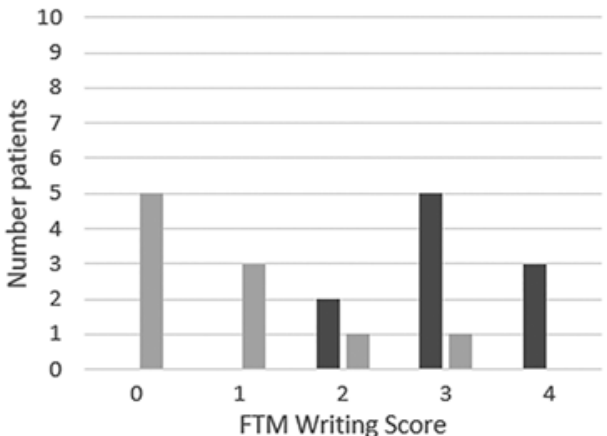

FTM Drinking Scores for 1st GKT

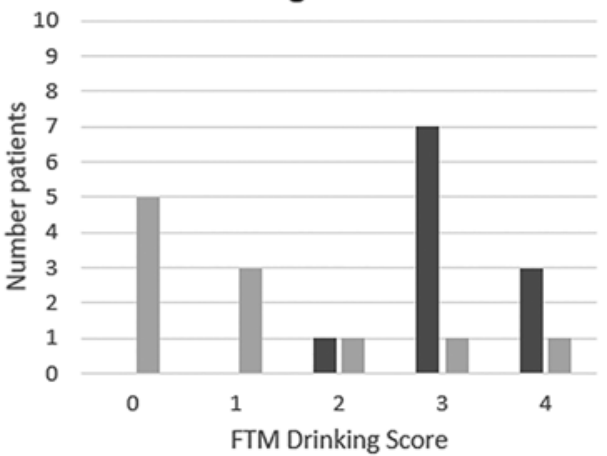

FTM Drawing Scores for 2nd GKT

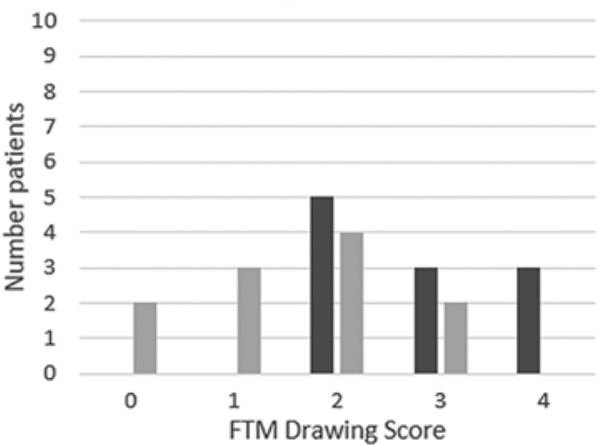

apreop

a postop

FIG. 3. A: Comparison of right hand FTM tremor, writing, drawing, and drinking scores obtained before and after first GKT. All 4 FTM values showed statistically significant improvements after first GKT. B: Comparison of left hand FTM Tremor, drawing, and drinking scores obtained before and after second GKT. All 3 FTM values showed statistically significant improvements after second GKT.

and staged bilateral RFT both represent important and well-reported options. Although bilateral DBS and staged RFT have each been noted to provide early benefit, over time that benefit wanes in many patients. ${ }^{26,30}$ Magnetic resonance imaging-guided focused ultrasound (MRgFUS) is another minimally invasive technique for management 


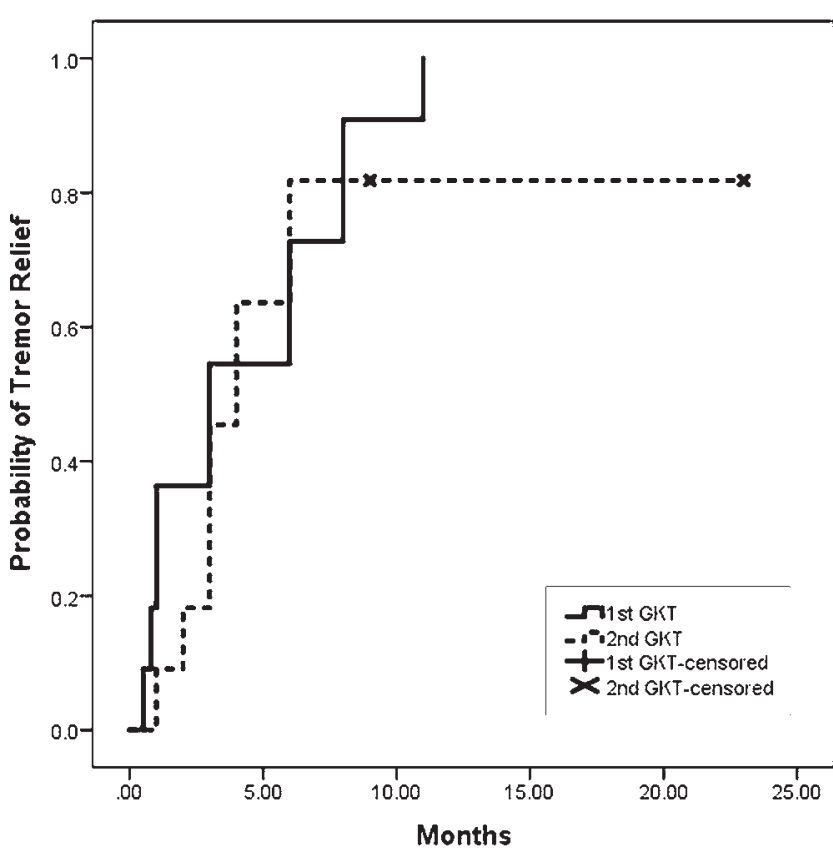

FIG. 4. Kaplan-Meier curve showing time to onset of tremor relief after first (solid line) and second (dotted line) GKT.

of medically refractory ET. ${ }^{7}$ Initial encouraging results of unilateral ET thalamotomy using MRgFUS have been reported, but there are no data available for staged bilateral thalamic lesions. ${ }^{3,7,17,31}$ GKT is a minimally invasive treatment that has a longer delay in onset of tremor relief but provides long-lasting relief of tremor. Bilateral GKT may be the only treatment option for patients with medically refractory bilateral tremor who are not eligible for invasive DBS or RFT approaches. Despite almost 50 years of experience with the creation of stereotactic thalamic lesions using the Leksell Gamma Knife, the role of bilateral GKT is still considered controversial. Advocates for GKT report sustained clinical benefit and a low risk of treatment-related AREs. 4,16,20,21,32,37

ET patients who undergo GKT experience tremor reduction in the contralateral arm. However, PD patients treated with GKT noted statistically significant improvement in rigidity as well. ${ }^{21}$ Some ET patients undergoing GKT noted qualitative improvements in neck, jaw, and head tremor. ${ }^{15}$ The expansion of benefit to include tremor in other areas of the body further defines the robust nature of GKT. Since patients with neurodegenerative diseases (i.e., PD and MS) may experience disease progression with worsening tremor in the untreated arm, we believe that staged bilateral GKT is both useful and underutilized for patients ineligible for more invasive DBS or RFT procedures.

Even though staging bilateral GKT procedures with a 1-year interval seems to allow sufficient time to assess benefit as well as adverse effects, long-term concerns related to radiosurgery thalamotomy include the potential dysphagia, dysarthria, speech disorders, and cognitive deficits. ${ }^{2,4,10,24}$ Prior reports describe 2 delayed deaths attributed to bilateral GKT. One patient died following bilateral high-dose (160 Gy and $180 \mathrm{GY})$ medial thala- motomy for chronic pain. ${ }^{34}$ To expand the lesion volume, a 2 -isocenter technique was used on both sides. The patient died 12 months after the second lesion was created, and postmortem examination revealed thalamic and brainstem radiation necrosis. A second patient, suffering from PD, died after bilateral high-dose GKT (200 Gy to 1 side followed by 150 Gy to the opposite thalamic target 8 months later). ${ }^{24}$ One month after the second GKT, the patient developed progressive dysarthria, dysphagia, gait ataxia, and aspiration pneumonia leading to death. These case reports highlight the importance of the selection of an appropriate radiobiological dose (130-140 Gy) using a single 4-mm isocenter and separating the staging by 12 months or more.

\section{Implications of the Imaging Changes}

Overall, in our case series, 6 patients showed appropriate imaging changes (Type A response), 1 showed streaking along the IC on T2-weighted FLAIR images (Type B response), and 1 showed an unexpectedly large lesion volume and significant perilesional edema on the short-term imaging follow-up studies (so-called super-responder). Similar imaging changes were reported by Ohye et al. ${ }^{21,23}$ We noted that such imaging changes stabilized between 1 and 2 years. After 4-5 years, the enhancement completely resolved, but a stable residual VIM T1 lesion was noted, without associated T2 or FLAIR signal changes. A single patient with MS-related tremor developed a super-responder lesion at 8 months (10-mm rim-enhancing lesion with significant reactive changes and streaking along the IC) after first GKT using a 130-Gy central dose. The second GKT was delayed until the initial imaging changes resolved. Because this patient was considered a super-responder the central dose for second GKT was lowered to $125 \mathrm{~Gy}$.

\section{Outcomes of This Study}

After the first GKT, all patients experienced longlasting benefit. FTM scores (tremor, handwriting, drawing, and drinking) decreased more than 2 points on average. There was significant improvement in tremor and functional capability of the dominant hand. Eight patients (72.7\%) experienced tremor arrest or barely perceptible tremor. Following the second GKT, contralateral FTM scores (tremor, drawing, and drinking) decreased without ARE or AE. While these improvements were also longlasting, they were less dramatic than those following the first GKT (Table 2).

Such differences in improvement could be due to lower initial FTM scores for the second GKT (due to small ipsilateral benefit from first GKT). However, these improvements could also be due to a higher dose for first GKT in comparison with second GKT. Specifically, for the first GKT the median dose was 140 Gy (range 130-200 Gy), while for the second GKT the median dose was 130 Gy (range 125-140 Gy). As an example, a patient with PD had complete tremor arrest and complete resolution of writing, drawing, and drinking difficulties starting 8 months after a first GKT. This improvement was maintained at last follow-up. Surprisingly this same patient underwent a second GKT (right VIM, 120 Gy) and as of the last followup had sustained no additional tremor improvement (23 


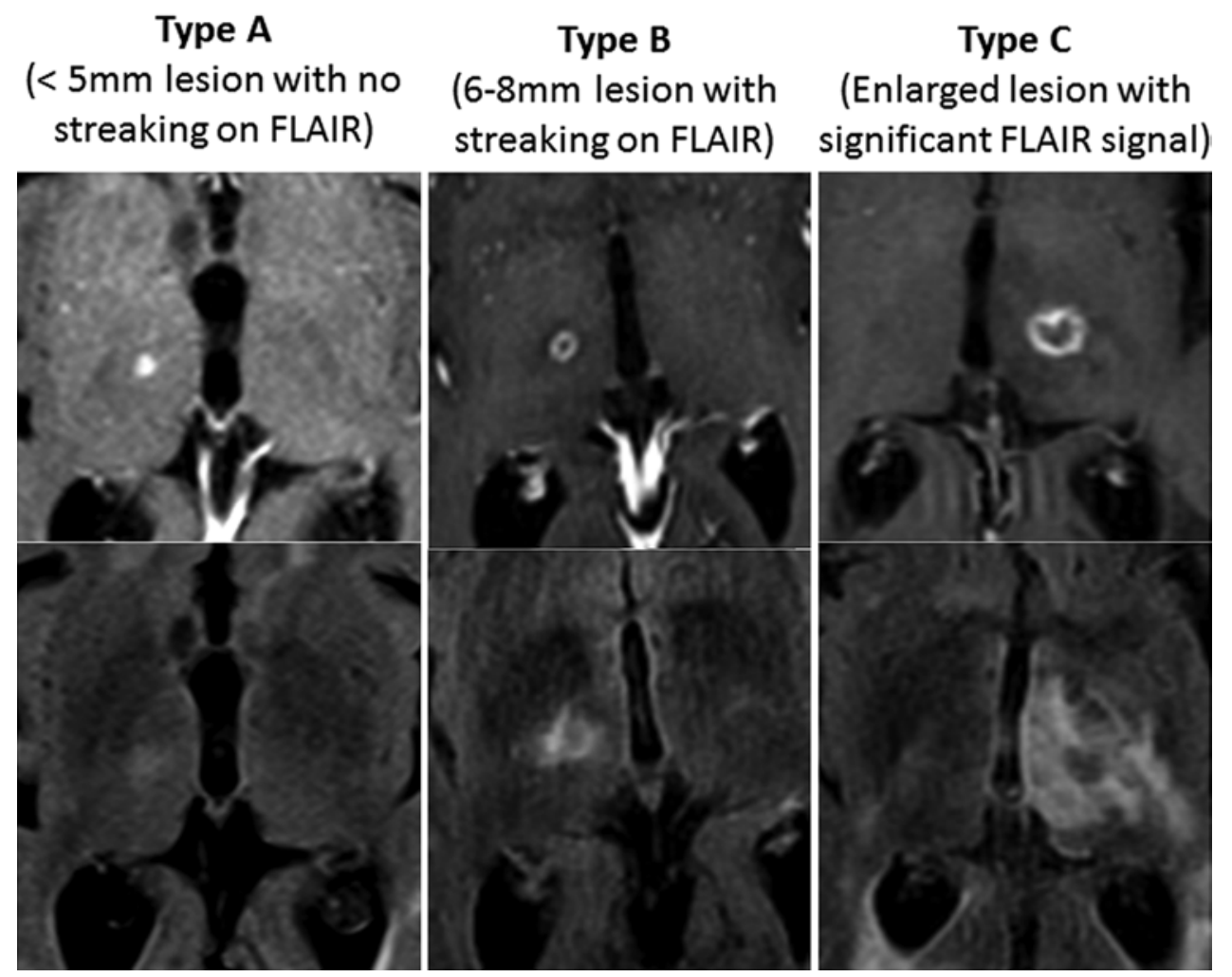

FIG. 5. Figure showing 3 types of imaging changes between 4 and 8 months after GKT. Type $A(\leq 5-m m$ enhancing lesion on T1-weighted contrast-enhanced images with no streaking along IC on T2-weighted FLAIR images) was seen in 6 of 8 patients for whom short-term MRI follow-up was available. Type B (6- to 8-mm enhancing lesion on T1-weighted contrast-enhanced images with streaking along IC on T2-weighted FLAIR images) was seen in 1 patient. Type $C$ (> 10-mm enhancing lesion on T1-weighted contrast-enhanced images with significant signal hyperintensity on T2-weighted FLAIR images) was seen in 1 patient.

months post-GKT). While this lack of improvement could also be due to a slight change in target coordinates, it is more likely due to the decreased central dose. In another patient, who had ET, the first GKT (left VIM, 130 Gy) led to moderate tremor decrease starting 6 months post-GKT. After the second GKT (right VIM, 140 Gy), the same patient had tremor arrest within 2 months post-GKT.

Overall, in this relatively small experience we could detect no treatment-related variables (such as age, diagnosis, or dose) that correlated with the clinical outcome. The outcomes of the current study are likely due to an appropriately rigid protocol for patient selection, more than 12 months between stages, and use of the target dose recently confirmed by Ohye et al. as effective with low risk..$^{21,23} \mathrm{Of}$ note, no patients experienced any long-term clinical ARE or AE from either the first or second GKT.

Since 1987, more than 14,000 patients have undergone Gamma Knife stereotactic radiosurgery at the Center for Image-Guided Neurosurgery at the University of Pittsburgh Medical Center for treatment of vascular, neoplastic, and functional brain disorders. Thalamic radiosurgery represents only $0.5 \%$ of all cases, and bilateral GKT represents only $5 \%$ of all thalamic radiosurgery procedures for tremor. Based on the encouraging outcomes of this study and our experience with Gamma Knife radiosurgery, we believe that bilateral GKT is a currently underutilized surgical procedure that can be safe and effective for carefully selected patients with bilateral tremor. Such patients are generally ineligible for more invasive tremor surgery because of associated medical comorbidities or the need for chronic anticoagulation therapy.

\section{Neurological Adverse Events of Bilateral Thalamic Procedures}

Previous studies of bilateral GKT, bilateral DBS, and bilateral RFT have raised concerns of AEs such as dysphagia, dysarthria, and ataxia. ${ }^{4-6,11,12,14,19,24-30,33}$ In a series of reports, Duma and colleagues described 4 PD patients who underwent staged bilateral GKT (6 months apart) via a single 4-mm collimator. ${ }^{4-6}$ One patient experienced dysarthria (starting 1 week post-GKT) that was maintained through the last follow-up. Ohye et al. ${ }^{21}$ reported on 5 patients who underwent bilateral GKT (4-mm collimator) roughly 1 year apart.

Okun et al. ${ }^{24}$ reported that 2 patients (total number of patients not reported) experienced AEs from bilateral GKT. One patient had hypophonia after bilateral GKT (200 Gy each, 2 months apart). Another patient experienced dysphagia and subsequently died after bilateral GKT (200 Gy, 150 Gy, 8 months apart). While the AEs and the death are unfortunate, these patients underwent bilateral GKT with higher doses and shorter time intervals than those employed in the present study. Young et al. ${ }^{36}$ reported on 42 ET patients who underwent bilateral GKT with a mean 
interval of 20 months (range 12-65 months) between procedures. Patients only underwent a second GKT if the first GKT was effective without AE. No patient reported any AE from the second GKT.

Bilateral DBS is an important management option, especially for younger healthy patients with medically refractory bilateral tremor. Pahwa et al. ${ }^{26}$ studied 16 patients (12 with follow-up) who underwent bilateral DBS and reported that $88 \%$ and $75 \%$ of PD patients treated with bilateral DBS developed dysarthria and dysphagia, respectively. Plaha et al. ${ }^{27}$ reported that $30 \%-50 \%$ of patients treated with bilateral DBS suffer from dysarthria and disequilibrium, with many developing habituation to stimulation. Putzke et al. ${ }^{28}$ reported that $27 \%$ of patients treated with bilateral DBS developed dysarthria. Huss et al. ${ }^{11}$ reported that 35 of 57 patients treated with bilateral DBS experienced AEs, most notably dysarthria and gait instability. Ondo et al. ${ }^{25}$ found that 16 of 21 patients treated with bilateral DBS experienced complications, most notably dysarthria and gait imbalance.

Bilateral RFT is another option for medically refractory bilateral tremor; however, it is also associated with significant AEs. Kelly et al. ${ }^{14}$ studied 24 patients treated with staged bilateral RFT (within 4 years). Twenty-one patients had reduced voice volume before the second RFT, and 9 patients had further reduction of voice volume following the second RFT. Moriyama et al. ${ }^{19}$ reported that 5 of 9 patients treated with bilateral RFT developed complications. These data suggest that while bilateral thalamic procedures may alleviate tremor, they are associated with significant AEs. In light of possible side effects, careful patient selection and appropriate timing of second thalamic procedure are warranted.

\section{Patient Selection for Bilateral GKT}

The majority of patents only require GKT for relief of dominant hand tremor. GKT for dominant hand tremor significantly improves their quality of life, as the dominant hand is used for most activities of daily living. However, a small percentage of tremor patients may require bilateral GKT to improve their activities of daily living. A second GKT should be considered for patients whose quality of life is significantly impacted by the tremor affecting the untreated side. Patients with head or body tremor can also considered for bilateral GKT. Patients with significant deterioration in quality of life due to progression of tremor on the untreated side are also reasonable candidates for bilateral GKT. Patients who underwent other bilateral procedures such as DBS but did not experience tremor relief are also candidates for bilateral GKT. In our series 1 patient had been unsuccessfully treated with bilateral DBS for bilateral tremor. There may also be some patients who require improvement in bilateral tremor in order keep their employment. In the present study we had 3 patients (a painter, a dentist, and a teacher) who required a second GKT because they could not perform their jobs without significant reduction in bilateral tremor. However, if a patient requests a second GKT because of significant improvement in contralateral tremor from a first GKT, patient counseling and declining to perform GKT are always options if the surgeon feels that patient is able to carry out activities of daily living and does not believe that improvement in the tremor in the contralateral side will significantly improve their quality of life.

\section{Limitations}

This was a retrospective study of the outcomes of bilateral staged GKT for patients ineligible for DBS or RFT, and our outcomes likely reflect the effect of patient selection bias.

\section{Final Thoughts}

Importantly, there is no published randomized control trial for bilateral GKT, bilateral DBS, and bilateral RFT. Interestingly, some authors have noted hybrid procedures in the literature. ${ }^{9,23}$ Specifically, Franzini et al. ${ }^{9}$ noted 2 patients with bilateral PD, who were treated with DBS on one side and CyberKnife on other side. Both patients experienced bilateral tremor control without AE. Ohye et al. ${ }^{23}$ described 9 patients who were treated with RFT on 1 side and then GKT on the other side. MRI-guided focused ultrasound (MRgFUS) is another minimally invasive technique that is being used for unilateral or bilateral tremor. ${ }^{7}$ It would be interesting to see the results and evaluate the potential of hybrid procedures to expand the future literature.

\section{Conclusions}

Overall, 11 patients (100\%) experienced benefit after the first GKT, and 9 patients $(81.8 \%)$ experienced benefit after the second GKT. No patient experienced diminished tremor relief or AEs following the second GKT. Based on these encouraging long-term outcomes, we believe that staged bilateral GKT should be considered for carefully selected patients suffering from medically refractory bilateral tremor. Such patients generally are considered ineligible for more invasive procedures such as DBS.

\section{References}

1. Baumann CR: Epidemiology, diagnosis and differential diagnosis in Parkinson's disease tremor. Parkinsonism Relat Disord 18 (Suppl 1):S90-S92, 2012

2. Campbell AM, Glover J, Chiang VL, Gerrard J, Yu JB: Gamma Knife stereotactic radiosurgical thalamotomy for intractable tremor: a systematic review of the literature. Radiother Oncol 114:296-301, 2015

3. Chang WS, Jung HH, Kweon EJ, Zadicario E, Rachmilevitch I, Chang JW: Unilateral magnetic resonance guided focused ultrasound thalamotomy for essential tremor: practices and clinicoradiological outcomes. J Neurol Neurosurg Psychiatry 86:257-264, 2015

4. Duma CM: Movement disorder radiosurgery-planning, physics and complication avoidance. Prog Neurol Surg 20:249-266, 2007

5. Duma CM, Jacques D, Kopyov OV: The treatment of movement disorders using Gamma Knife stereotactic radiosurgery. Neurosurg Clin N Am 10:379-389, 1999

6. Duma CM, Jacques DB, Kopyov OV, Mark RJ, Copcutt B, Farokhi HK: Gamma Knife radiosurgery for thalamotomy in parkinsonian tremor: a five-year experience. J Neurosurg 88:1044-1049, 1998

7. Elias WJ, Huss D, Voss T, Loomba J, Khaled M, Zadicario E, et al: A pilot study of focused ultrasound thalamotomy for essential tremor. N Engl J Med 369:640-648, 2013 
8. Fahn S, Tolosa E, Marin C: Clinical Rating Scale for Tremor, ed 2. Baltimore: William \& Wilkins, 1993

9. Franzini A, Marchetti M, Brait L, Milanesi I, Messina G, Forapani E, et al: Deep brain stimulation and frameless stereotactic radiosurgery in the treatment of bilateral parkinsonian tremor: target selection and case report of two patients. Acta Neurochir (Wien) 153:1069-1075, 2011

10. Frighetto L, Bizzi J, Annes RD, dos Santos Silva R, Oppitz P: Stereotactic radiosurgery for movement disorders. Surg Neurol Int 3 (Suppl 1):S10-S16, 2012

11. Huss DS, Dallapiazza RF, Shah BB, Harrison MB, Diamond J, Elias WJ: Functional assessment and quality of life in essential tremor with bilateral or unilateral DBS and focused ultrasound thalamotomy. Mov Disord 30:1937-1943, 2015

12. Jankovic J, Cardoso F, Grossman RG, Hamilton WJ: Outcome after stereotactic thalamotomy for parkinsonian, essential, and other types of tremor. Neurosurgery 37:680-687, 1995

13. Karnofsky DA, Burchenal JH: The clinical evaluation of chemotherapeutic agents in cancer, in Macleod CM (ed): Evaluation of Chemotherapeutic Agents. New York: Columbia University Press, 1949

14. Kelly PJ, Gillingham FJ: The long-term results of stereotaxic surgery and L-dopa therapy in patients with Parkinson's disease. A 10-year follow-up study. J Neurosurg 53:332-337, 1980

15. Kondziolka D, Ong JG, Lee JY, Moore RY, Flickinger JC, Lunsford LD: Gamma Knife thalamotomy for essential tremor. J Neurosurg 108:111-117, 2008

16. Kooshkabadi A, Lunsford LD, Tonetti D, Flickinger JC, Kondziolka D: Gamma Knife thalamotomy for tremor in the magnetic resonance imaging era. J Neurosurg 118:713-718, 2013

17. Lipsman N, Schwartz ML, Huang Y, Lee L, Sankar T, Chapman M, et al: MR-guided focused ultrasound thalamotomy for essential tremor: a proof-of-concept study. Lancet Neurol 12:462-468, 2013

18. Louis ED, Ottman R: How many people in the USA have essential tremor? Deriving a population estimate based on epidemiological data. Tremor Other Hyperkinet Mov (N Y) 4:259, 2014

19. Moriyama E, Beck H, Miyamoto T: Long-term results of ventrolateral thalamotomy for patients with Parkinson's disease. Neurol Med Chir (Tokyo) 39:350-357, 1999

20. Niranjan A, Kondziolka D, Baser S, Heyman R, Lunsford LD: Functional outcomes after Gamma Knife thalamotomy for essential tremor and MS-related tremor. Neurology 55:443-446, 2000

21. Ohye C, Higuchi Y, Shibazaki T, Hashimoto T, Koyama T, Hirai T, et al: Gamma Knife thalamotomy for Parkinson disease and essential tremor: a prospective multicenter study. Neurosurgery 70:526-536, 2012

22. Ohye C, Shibazaki T, Hirato M, Inoue H, Andou Y: Gamma thalamotomy for parkinsonian and other kinds of tremor. Stereotact Funct Neurosurg 66 (Suppl 1):333-342, 1996

23. Ohye C, Shibazaki T, Sato S: Gamma Knife thalamotomy for movement disorders: evaluation of the thalamic lesion and clinical results. J Neurosurg 102 Suppl:234-240, 2005

24. Okun MS, Stover NP, Subramanian T, Gearing M, Wainer BH, Holder CA, et al: Complications of Gamma Knife surgery for Parkinson disease. Arch Neurol 58:1995-2002, 2001

25. Ondo W, Almaguer M, Jankovic J, Simpson RK: Thalamic deep brain stimulation: comparison between unilateral and bilateral placement. Arch Neurol 58:218-222, 2001

26. Pahwa R, Lyons KE, Wilkinson SB, Simpson RK Jr, Ondo WG, Tarsy D, et al: Long-term evaluation of deep brain stimulation of the thalamus. J Neurosurg 104:506-512, 2006

27. Plaha P, Patel NK, Gill SS: Stimulation of the subthalamic region for essential tremor. J Neurosurg 101:48-54, 2004

28. Putzke JD, Uitti RJ, Obwegeser AA, Wszolek ZK, Wharen RE: Bilateral thalamic deep brain stimulation: midline tremor control. J Neurol Neurosurg Psychiatry 76:684-690, 2005

29. Speelman JD, Schuurman R, de Bie RM, Esselink RA, Bosch DA: Stereotactic neurosurgery for tremor. Mov Disord 17 (Suppl 3):S84-S88, 2002

30. Tseng HM, Su PC, Liu HM, Liou HH, Yen RF: Bilateral subthalamotomy for advanced Parkinson disease. Surg Neurol 68 (Suppl 1):S43-S51, 2007

31. Wintermark M, Huss DS, Shah BB, Tustison N, Druzgal TJ, Kassell N, et al: Thalamic connectivity in patients with essential tremor treated with MR imaging-guided focused ultrasound: in vivo fiber tracking by using diffusion-tensor MR imaging. Radiology 272:202-209, 2014

32. Witjas T, Carron R, Krack P, Eusebio A, Vaugoyeau M, Hariz $\mathrm{M}$, et al: A prospective single-blind study of Gamma Knife thalamotomy for tremor. Neurology 85:1562-1568, 2015

33. Yamamoto T, Katayama Y, Kano T, Kobayashi K, Oshima H, Fukaya C: Deep brain stimulation for the treatment of parkinsonian, essential, and poststroke tremor: a suitable stimulation method and changes in effective stimulation intensity. J Neurosurg 101:201-209, 2004

34. Young RF: Functional neurosurgery with the Leksell Gamma Knife. Stereotact Funct Neurosurg 66:19-23, 1996

35. Young RF, Jacques S, Mark R, Kopyov O, Copcutt B, Posewitz A, et al: Gamma Knife thalamotomy for treatment of tremor: long-term results. J Neurosurg 93 (Suppl 3):128135,2000

36. Young RF, Li F, Vermeulen S, Meier R: Gamma Knife thalamotomy for treatment of essential tremor: long-term results. J Neurosurg 112:1311-1317, 2010

37. Young RF, Vermeulen SS, Grimm P, Posewitz A: Electrophysiological target localization is not required for the treatment of functional disorders. Stereotact Funct Neurosurg 66 (Suppl 1):309-319, 1996

\section{Disclosures}

Dr. Lunsford reports a consultant relationship with Elekta $\mathrm{AB}$ and DBMB and direct stock ownership in Elekta AB.

\section{Author Contributions}

Conception and design: Niranjan. Acquisition of data: Raju. Analysis and interpretation of data: Niranjan, Raju. Drafting the article: Niranjan, Raju. Critically revising the article: Niranjan, Monaco, Flickinger, Lunsford. Reviewed submitted version of manuscript: Niranjan, Monaco, Flickinger, Lunsford. Approved the final version of the manuscript on behalf of all authors: Niranjan.

\section{Correspondence}

Ajay Niranjan, Department of Neurological Surgery, University of Pittsburgh, Ste. B-400, UPMC, Presbyterian, PA 15213. email: niranjana@upmc.edu. 